\title{
DISCUSSION
}

\section{A new analysis procedure to explain a slope failure at the Martin Lake mine}

\author{
T. W. MILLER and J. M. HAMILTON. (1989) Géotechnique 39, No. 1, 107-123
}

\section{R. L. Michalowski, University of Minnesota}

The Authors utilize the upper bound (kinematical) approach of limit analysis to evaluate the stability of slopes, taking into account the pore pressure in the soil mass. They have developed a failure mechanism which includes both rigid rotation and shear deformation (plane strain). However, I cannot agree with the Authors' method of calculations. In particular, it will be argued that the calculations of the energy dissipation rate in the plastic deformation process are incorrect.

The Authors consider soil whose skeleton obeys the Mohr-Coulomb yield condition and the flow rule associated with this condition. Accordingly, the yield condition is expressed in terms of the effective stress tensor components $\sigma_{\mathrm{ij}}{ }^{\prime}$

$$
f\left(\sigma_{\mathrm{ij}}\right)=0
$$

and the associative flow rule

$$
\dot{\varepsilon}_{i j}=\lambda\left(\partial f / \partial \sigma_{i j}{ }^{\prime}\right), \quad \lambda \geqslant 0
$$

defines the rate of deformation of the skeleton $\dot{\varepsilon}_{\mathrm{ij}}$ under the effective stress state satisfying equation (38). In the process of plastic deformation of the soil skeleton, the rate of energy (or work) dissipation per unit volume is given by the dot product of the effective stress tensor and the strain rate tensor

$$
\dot{D}=\sigma_{\mathrm{ij}}{ }^{\prime} \dot{\varepsilon}_{\mathrm{ij}}
$$

(see e.g. Atkinson, 1981). The Authors however, propose calculating the energy dissipation rate for saturated soil as the dot product of the total stress tensor, $\sigma_{\mathrm{ij}}=\sigma_{\mathrm{ij}}{ }^{\prime}+\delta_{\mathrm{ij}} p$ ( $p$ being the pore pressure), and the strain rate tensor of the skeleton, arriving at the following formula (equation (8) of the Paper)

$$
D_{\mathrm{v}}=\sigma_{\mathrm{ij}} \dot{\varepsilon}_{\mathrm{ij}}=\sigma_{\mathrm{ij}}{ }^{\prime} \dot{\varepsilon}_{\mathrm{ij}}+p \dot{\varepsilon}_{\mathrm{ii}}
$$

The second term in equation (41) represents the dot product of the pressure in water and the deformation rate of the skeleton, and has no specific mechanical interpretation. Water is assumed to be an incompressible and non-viscous liquid, therefore no energy is dissipated by the water. It could be argued that, during rapid deformation, energy might be dissipated owing to relative flow of water induced during the process of skeleton deformation (the skeleton being dilational, and the water incompressible). This, however, is a viscous effect (dependent on the deformation rate, negligible for low speeds), and beyond the capability of the classical limit analysis framework.

It was noticed by the Authors that the second term in their dissipation rate formula (equation (41)) reduces the total dissipation (for compressive pore pressure). The energy dissipation rate $D_{v}$, calculated according to that formula, would be negative for a cohesionless material. In such a case $D_{\mathrm{v}}$ would indicate the energy production rather than dissipation. Energy dissipation must be non-negative in plastic deformation processes (e.g. Hill, 1950). If the deformation process was to be analysed in terms of thermodynamics, the consequence of negative dissipative work would be a negative increment of entropy. This, however, would be in clear violation of the second law of thermodynamics (e.g. Ziegler, 1983). Equation (41) is then an incorrect way to calculate energy dissipation. The same criticism pertains to the Authors' calculations of energy dissipation along discontinuity surfaces (equation (30) of the referenced paper).

The technique suggested by the Authors for analysing the stability of slopes is inconsistent with the upper bound theorem which is used in the analysis. The normality rule (39) relates the strain rate of the skeleton to the effective stress, not the total stress. Consequently, if the total stress is used in calculations of work dissipation, the principle of maximum work cannot be proved true, and the theorems of limit analysis are no longer valid.

Increase of the water table in slopes adversely affects their stability, although this influence enters the limit analysis through the external forces work (buoyancy and seepage forces), not through the internal dissipation.

\section{Authors' reply}

The Authors appreciate the opportunity to discuss an alternative interpretation of how upper-bound analyses may be applied to 
effective-stress problems that involve arbitrary pore pressure distributions. Michalowski appears to be concerned that the analysis procedure presented in the Paper includes terms that it should not (i.e., energy dissipation due to pore pressures) and ignores terms that it should include (i.e., work done by seepage and buoyant forces). These terms are in fact the same, and the one term will have a positive or negative sign depending on where it appears in the equation used to determine the solution. We apologize for the confusion caused by presenting the concept of 'negative dissipation' as we did without a short discussion of alternative approaches. Such an oversight was due to the fact that after considering the alternative approaches, the one presented in the Paper was adopted because it simplified the calculations and was appealing from the physical point of view. After using this approach over a period of time, we neglected to consider that others may not have the same biases.

The primary argument by Michalowski is that the dissipation of internal energy should be calculated based on the effective stress rather than the total stresses. Further, it is pointed out that the contribution of pore pressures to slope instability enters only through buoyant and seepage forces. From these arguments, it is then concluded that the use of total stress in the dissipation calculation leads to a thermodynamically unreasonable situation of negative dissipation and that the principle of maximum work cannot be proved true. Thus the limit analysis theorems do not apply to the class of problems covered in the Paper. The implications of these arguments are that the results of the analyses in the Paper are not upper bounds and, of more importance, the procedure cannot be applied in general.

These assertions can be addressed in two different ways: one that avoids the issue and calls negative dissipation positive work and the other that shows (given the assumptions of a drained analysis') the basic principle behind the upperbound theorem to be valid whether energy dissipation is determined in terms of effective or total stresses. In the former case, one may regard pore pressures as either part of the system (internal dissipation) or external to it (seepage and buoyant forces) and generate identical governing equations. In the second case, it might be argued that energy dissipation is more naturally calculated from total stresses since it is the total stresses that are in equilibrium with the external loads. Regardless, the arguments in this second case reduce essentially to the same as in the first case.

In general, one formulates an upper-bound analysis by developing a kinematically admissible failure mechanism, equating the rates of internal energy dissipation and work done by boundary and body force, and then solving the equation to determine a generalized failure load. It is possible to calculate the internal dissipation of energy based only on the effective stresses and then to treat the terms $p \varepsilon_{\mathrm{ii}}$ from equation (41) as the negative of work done by body forces. In this case, the body forces are provided by the water pressure and work is done by a constant pressure acting to expand a dilating medium. Expansion of water is not required to do work because, within the context of a drained analysis, the pore pressures are prescribed as a function of space independent of the deformations.

It is reasonable to ask whether the work done by water pressures in a dilating medium-as determined from the integration of $p \varepsilon_{\mathrm{ii}}$ over the deforming volume-is equal to the work done by seepage and buoyancy forces. This can be shown to be true in general by the application of the divergence theorem as follows

$$
\begin{aligned}
\int p \varepsilon_{\mathrm{ii}} \mathrm{d} V & =\int\left(p u_{\mathrm{i}}\right), \mathrm{d} V-\int\left(p, u_{\mathrm{i}}\right) \mathrm{d} V \\
& =\int p u_{\mathrm{i}} v_{\mathrm{i}} \mathrm{d} S-\int\left(p, u_{\mathrm{i}}\right) \mathrm{d} V,
\end{aligned}
$$

where $u_{\mathrm{i}}$ is the $i$ th component of the velocity vector, the comma denotes differentiation with respect to $i$ th spatial direction, and $v_{\mathrm{i}}$ is the $i$ th component of the normal vector to the surface elements $\mathrm{d} S$. The summation convention on double subscripts is also assumed. After properly accounting for sign convections and direction of normals relative to the pressure and velocities, the surface and volume integral terms on the right hand side of equation (42b) can be recognized as the work done by the buoyant and seepage forces, respectively. Therefore, either approach results in an identical formulation; however, in the case where pore pressures are assumed to be internal to the system, only one equation needs to be solved. Alternatively, one could just solve the one equation and place it in the solution wherever it is felt it belongs.

Negative dissipation may be a troubling term to some. We feel, however, that the advantages of approaching the problem in this way outweigh this disadvantage. The occurrence of this term does not imply an overall entropy decrease because it is balanced by work done by other forces.

The simple consistency in the formulation that allows alternative physical interpretations certainly must be indicative of something concerning the fundamentals of the upper-bound formulation and that internal dissipation may be calculated based on either effective or total stresses as long as the strains and displacements are kinematically 
admissible. That this is so is demonstrated in the next paragraphs. Such a demonstration does not require a complete restatement of any of the various proofs of the upper-bound theorem, for example in Chen (1972). Essentially these proofs use virtual-work arguments to show that a set of external loads or body forces are upper bounds to a complete, plastic collapse solution. The essential argument that is required follows from Drucker's postulate, for example see Chen (1972).

Recall that the proofs define the (total) stresses that are associated with the complete plastic collapse solution as $\sigma$ and the (total) stresses that are consistent with the plastic strain rates in the assumed failure solution as $\sigma^{*}$. The plastic strain rates in the assumed failure solution are defined as $\varepsilon^{*}$. These strain rates are kinematically admissible if they are calculated from the appropriate yield condition, expressed in terms of the effective stresses. The key part of the proof depends on whether or not

$$
\left(\sigma^{*}-\sigma\right) \varepsilon^{*} \geqslant 0 \text {. }
$$

Now by the definition of the effective stress, it follows that

$$
\left(\sigma^{*}-\sigma^{\prime}\right) \varepsilon^{*}+\left(p^{*}-p\right) \varepsilon^{*} \geqslant 0 .
$$

Here, the primes denote effective stresses. In a drained analysis, the pore pressures are prescribed and are independent of the displacement field, therefore $p^{*}=p$. The remaining term in equation (44) is the essence of Drucker's postu- late, and the proof is valid for effective as well as total stresses.

For the purposes of the Paper in question, we chose to treat the pore pressure terms as internal dissipation because it appeared more natural and was simpler to treat then as part of the system. Others (i.e. Giam \& Donald, 1989), appear to agree. However, we do not object to treating pore pressures as external to the system and calculating appropriate terms as external work. To the Authors, it seems primarily a question of one's point of view. Regardless, as long as the contributions of the pore pressures are included properly, the solution will be a valid upper bound.

\section{ACKNOWLEDGEMENTS}

The Authors would like to acknowledge G. L. Bowers and J. D. Murff for their contributions in clarifying many of the concepts discussed.

\section{REFERENCES}

Atkinson, J. H. (1981). Foundations and slopes. An introduction to applications of critical state soil mechanics. New York: Wiley.

Giam, P. S. K. \& Donald I. B. (1989). Soil stability analysis by an upper bound plasticity method. Civil Engineering Research Report, Monash University.

Hill, R. (1950). The mathematical theory of plasticity. Oxford: Clarendon.

Ziegler, H. (1983). An introduction to thermodynamics. Amsterdam: North-Holland. 